\title{
Fuzzy modeling to 'understand' personal preferences of mHealth users: a case study
}

\author{
R.C.Y. Nuijten ${ }^{a}$, U. Kaymak ${ }^{a}$, P.M.E. Van Gorp ${ }^{a}$, \\ M. Simons ${ }^{b}$, P.E.W. Van den Berg ${ }^{c}$ and P.M. Le Blanc ${ }^{a}$ \\ ${ }^{a}$ Department of Industrial Engineering \& Innovation Sciences, Eindhoven University of Technology \\ $\{$ r.c.y.nuijten, u.kaymak, p.m.e.v.gorp, p.m.le.blanc\}@tue.nl \\ ${ }^{b}$ Department of Social Sciences, Wageningen University and Research \\ monique.simons@wur.nl \\ ${ }^{c}$ Department of the Built Environment, Eindhoven University of Technology \\ p.e.w.v.d.berg@tue.nl
}

\begin{abstract}
This case study evaluates to what extent personal preferences can be automatically derived from user event data in an mHealth setting. Based on a theoretical framework, user preferences are described using six classes. Based on this framework, a structure of six Takagi-Sugeno fuzzy inference systems was constructed and evaluated against baseline data from an official survey for measuring the framework's constructs. From this analysis, it was found that user preferences may be derived from user event data using fuzzy modeling with accuracy scores that are higher than a random predictor would typically achieve.
\end{abstract}

Keywords: fuzzy inference system, TakagiSugeno, personalization, mHealth.

\section{Introduction}

Several mHealth tools have proven to be efficient in engaging Humans in a variety of different health-related tasks [3, 6]. Although effective in the short-term, these interventions often fail to retain their impact in the long-term [7]. Hence, the challenge is to design mHealth tools that are on the long term sufficiently engaging. Tailoring an intervention to the exact needs and preferences of a user (i.e., personalization) may yield higher engagement [10], because - whenever one's preferences and needs are captured - effort can be directed efficiently to cater to these desires.

It is challenging to find the target of the personalization: of course, one's preferences may be learned from a questionnaire, but questionnaires are often perceived as cumbersome, and therefore might not be completed. Therefore, this study explores whether user preferences can be derived from user event data in a manner that surveys become less important. In this case study, user event data (e.g., the number of days users were online, as well as the number of views per page per user) of a particular mHealth tool is evaluated to learn individual user preferences. Fuzzy modeling techniques are employed because one's preferences may not be defined crisply. Moreover, one may have multiple (competing) preferences at the same time. These cases of granularity and imprecision may be handled especially well by fuzzy modeling techniques.

\section{Background}

\subsection{The mHealth tool}

Within this case study, the mHealth tool GameBus (see e.g., www.gamebus.eu) is evaluated. This tool was especially designed for health promotion, and provides a highly configurable gamification platform for supporting this purpose.

GameBus adopts gamification techniques ("the use of game design elements in non-game contexts" 4) to intrinsically motivate its users to engage with the application. According to the classification of gamification elements by Hamari, GameBus implements the gamification mechanisms of 'challenges', 'points', 'goals', 'progress', 'leaderboards', and 'rewards' 9]). These gamification mechanisms are implemented as follows: GameBus includes a mobile application that is designed to empower its end users to adopt a healthier lifestyle. The application tracks the (daily) healthy activities one performs, and rewards virtual points for these efforts. The number of virtual points awarded per activity depends on the particular challenges one chooses to take, since in a challenge, the rules for allocation of virtual points are defined. The platform is designed to enable the rewarding of any healthy activity: from social, to mental, to physical. For example, within a challenge, points can be awarded for running (physical activity) and performing acts of kindness (social activity) at the same time. Furthermore, one may choose to engage in these challenges individ- 
ually - comparing one's performance (i.e., the number of virtual points obtained) to a pre-defined goal - or together with close relatives - comparing one's performance against each other, or to other teams of related individuals. Moreover, GameBus provides features for social support: users can 'like' and comment on each other's healthy achievements, as well as they can chat with each other. Summarizing, GameBus allows users to perform the activities one enjoys truly as an individual in such a way that one is part of an integrated social interaction.

In order to claim points, users have to prove that they engaged in a specific healthy activity. This "proof of activity" comes from data that is either provided by external applications (i.e., a third-party step counter tracking the user's daily number of steps), or the GameBus application itself (i.e., a photograph of the user doing physical exercise in the gym). Hence, the application connects with existing applications, and provides a platform to translate inputs from other applications into a personal score, depending on dynamic game rules.

In this particular context of gamification and gameful systems, personalization often includes decisions on what particular content - e.g., what game design elements such as leaderboards, rewards, or challenges to confront a user with. As described before, user's preferences for a particular type of content may differ from person to person [14]. Hence, when making a decision in the context of a gameful system on confronting a user with a particular game design element or not, a decision-maker should ideally understand the user's preferences, to maximize her chances of striking the right chord. Within GameBus, personalization based on user preferences may be the implementation of personally tailored challenges, custom supportive messages, personalized rewards, etc.

\subsection{The framework for modeling user preferences}

To understand someone's personal preferences, several frameworks already exist, e.g., the Big Five Personality Traits framework [8. Also in the context of gamification and gameful systems, several mappings between user preferences and actual (game) content are already available, see for example Bartle's player types 11, or the HEXAD framework [11, 14].

In this case study, the HEXAD framework has been selected as theoretical foundation, since both this framework and the mHealth tool are based on the theory of gamification 4. Additionally, both the framework and the application are founded on self-determination theory [12. In self-determination theory it is proposed that Human behavior is determined by the interplay of three basic psychological needs: autonomy, competence, and relatedness [12]. Autonomy refers to the desire to self-organize experience and behavior. Competence is the need for challenge and the experience of one's effectiveness. Relatedness refers to the need to experience connections to other people.

The HEXAD model identifies six types of users: 1) Socialisers enjoy interacting with others and creating social connections; 2) Free spirits want to create and explore; 3) Achievers are looking for ways to learn new things and improve themselves; 4) Philanthropists want to enrich the lives of others in some way with no expectation of reward; 5) Players will do what is needed of them to collect rewards from a system; and 6) Disruptors want to disrupt a system, either directly or through others to force positive or negative change.

\section{Methodology}

The main goal of this case study is to evaluate whether user preferences can be understood from user event data. In this case, the user's degree of belonging to the different HEXAD classes - which are established from an official survey 14 - are assumed to be the ground truth for one's actual preferences. The challenge is to accurately predict these membership degrees, based on parameters that are derived from the user's event data that is available from the mHealth tool GameBus.

\subsection{Data collection}

Within this study, data is evaluated that was gathered using GameBus during a health promotion campaign at Eindhoven University of Technology. During this campaign, a virtual competition was hosted among members of the departments of the university. Participants were invited to demonstrate their own department as the healthiest of the entire organization. To prove the 'health status' of their departments, participants were requested to track their daily, healthy activities - such as active transport, sports, and fruit intake - using GameBus. GameBus would then award virtual points for each activity, and the health status of a particular department would then be calculated as the average number of virtual points scored within that department. During a four-week period, participants were allowed to claim virtual points for their departments. To review their progress, GameBus allowed participants to compare their team performance (i.e., performance of their departments) and individual performance within their team. The goal of the competition was to obtain the highest score as a team. Moreover, for a scientific experiment, some participants were given a personal goal of obtaining at 
least 50 virtual points, before being allowed to claim a tangible reward (i.e., a mug).

From the user event data that was gathered by GameBus during this campaign, a total of 14 input parameters were derived. These parameters are:

$x_{1}$ : the number of points a subject obtained, $x_{2}$ : the subject's degree of surpassing her goal of obtaining 50 points, which is defined as:

$$
x_{2}=\left\{\begin{array}{ll}
0 & \mathrm{x}_{1}<50 \\
50 / x_{1} & \mathrm{x}_{1} \geq 50
\end{array},\right.
$$

$x_{3}$ : the number of activities a subject performed,

$x_{4}$ : the number of unique activity types a subject performed,

$x_{5}$ : the average number of activities a subject performed per days active,

$x_{6}$ : the number of supports utterances to other subjects,

$x_{7}$ : the number of reactions on other subjects' activities,

$x_{8}$ : the number of chat messages sent,

$x_{9}$ : the number of times a subject peeked at the leaderboard,

$x_{10}$ : the number of times a subject checked her activities,

$x_{11}$ : the number of times a subject glimpsed at the newsfeed,

$x_{12}$ : the number of times a subject looked up her team mates,

$x_{13}$ : the number of times a subject checked her profile,

$x_{14}$ : the number of times a subject peeked at other one's profiles.

Certain combinations of these input parameters were used to predict a subject's degree of belonging to each of the six classes as identified in the HEXAD framework (i.e., Socializer, Achiever, etc.). The subject's actual degree of belonging to these classes was obtained from post-test questionnaire responses, according to the survey by Tondello and colleagues [14]. In this questionnaire, subjects were asked to rate how well each survey item described them on a 7-point Likert scale. The survey included for example items such as "It is important to me to always carry out my tasks completely" to measure one's degree of belonging to the Achiever class, as well as items such as "It is important to me to feel like I am part of a community" to measure one's degree of belonging to the Socialiser class. To determine one's degree of belonging to a particular HEXAD class, the scores of the items corresponding to that class were summed separately.

\subsection{Data preparation}

After data was obtained via SQL, two datasets - an unsupervised dataset, including the values of input parameters for all campaign participants, as well as a (smaller) supervised dataset, including both the values of input parameters and the actual degree of belonging to the HEXAD classes for all campaign participants that completed the post-test survey - were prepared using Google Spreadsheets. In both datasets, minmax normalization was applied to each of the input variables $x_{1}$ to $x_{8}$. Hence, for these variables it was assumed that all participants kept the same mental model of granularity. In other words, it was assumed that any value of a particular variable would be judged the same membership to a particular condition - e.g., low, medium, or high - by all participants.

Furthermore, in both datasets, variables $x_{9}$ to $x_{14}$ were normalized per subject by dividing each separate element by the sum of these six elements per subject. Hence, these variables were normalized relative to each other, which implies that participants may have kept different mental models of granularity. In other words, it was implied that any value of a particular variable would be judged a different membership to a particular condition by different participants, depending on a subject's total number of page visits.

Finally - only for the supervised dataset - also the vector of actual measured HEXAD classes was normalized by dividing each separate degree of belonging by a subject's total degree of representation by the HEXAD framework (i.e., the sum of individual HEXAD class scores).

\subsection{Model building}

For each of the six HEXAD classes, a Takagi-Sugeno fuzzy inference system with at most 14 input parameters was derived, as described by Sousa and Kaymak 2]: Step 1 describes how the membership functions of input parameters were derived. Step 2 and Step 3 describe how a set of fuzzy rules were derived for each of the six classes. Finally, Step 4 describes how performance measures for these rules were derived. The software to perform these steps was created in Python (version 3.6.4), and is available via WwW.github.com/Louar/EUSFLAT-mHealth-FIS. git. This study adopts a first-order Takagi-Sugeno fuzzy modeling approach, since they have a large expressive capacity and can model complex non-linear mappings with a relatively small number of rules, compared to e.g., Mamdani fuzzy systems 2 .

\section{Step 1: Derive membership functions}

First, membership functions for the input variables are derived from the unsupervised dataset. Three- 
condition (i.e., low, medium, high), triangular membership functions were derived using "box plot parameters" (i.e., minimum, median and maximum) [5], see the upper graph in Figure 1 .
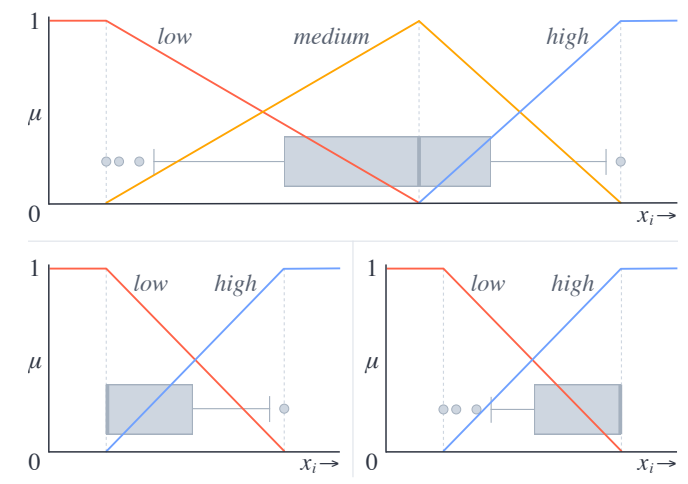

Figure 1: Transformation of box plot parameters to fuzzy membership functions.

Note that, whenever the minimum and median of an input variable were equivalent, only two membership functions were fitted (i.e., the membership function for the condition medium was omitted), see the bottom left graph in Figure 1. Similarly, whenever the median and maximum of an input variable were equivalent, the membership function for the condition medium was omitted, see the bottom right graph in Figure 1 .

\section{Step 2: Derive rule antecedents}

A fuzzy inference system consists of a rule base, that consists of a set of fuzzy IF-THEN rules. A fuzzy IFTHEN rule has two parts: an IF-part, which holds the rule's antecedents, and a THEN-part, which determines the consequence of a rule. The antecedents in the IF-part are combinations of input parameters and their conditions. For example, when the IF-part of a rule contains only one parameter - say $x_{1}$, which may have the conditions low, medium, and high then there are three possible variants (i.e., potential rules) of this IF-part: $x_{1}=$ low, $x_{1}=$ medium, and $x_{1}=$ high.

To derive these antecedents for each HEXAD class, it is necessary to understand what input parameters may influence - in other words predict - the degree of belonging to that particular class. This mapping of input parameters to HEXAD classes based on their predictive power was performed by the authors. The authors would be eligible to create this mapping since they are both the designers and developers of the mHealth tool under investigation - i.e., GameBus - and are familiar with the HEXAD framework. Summarizing, a set of relevant variables $\boldsymbol{R} \boldsymbol{V}_{\boldsymbol{o}}$ was derived for each HEXAD class $o$. Note that the number of elements in a set $\boldsymbol{R} \boldsymbol{V}_{\boldsymbol{o}}$ can never exceed 14, since only 14 input parameters are available in this study, see Section 3.1. However, a specific variable may occur in several sets of relevant variables for different HEXAD classes, because a particular variable may relate to multiple classes.

Given the mapping of input parameters $\boldsymbol{R} \boldsymbol{V}_{\boldsymbol{o}}$ to the HEXAD classes based on their predictive power, a set of antecedents may be derived (e.g., one's degree of belonging to the Achiever class is explained by her number of points obtained $\left(x_{1}\right)$, and number of peeks at the leaderboard $\left(x_{9}\right)$, and ... etc.). It is assumed that a rule's antecedents always consist of all the input parameters that are mapped onto the HEXAD class that a rule should explain. Different variants of this static combination of input variables exist in that an input variable can occur as an antecedent enforcing either of its at most three conditions (i.e., low, medium, high). Recall from the previous Step that some input parameters may lack a low or high condition, depending on the variable's particular box plot parameters.

Summarizing, a first-order Takagi-Sugeno system is constructed for each HEXAD class $o$, such that the rule base is formed by taking a grid partition with all combinations of fuzzy sets defined on the variables in $\mathbf{R V}_{o}$.

Subsequently, the different antecedents that constitute a rule are combined using Zadeh's AND operator [15]. Whenever it is assumed that one's degree of belonging to the HEXAD Achiever class is well predicted by input parameters $x_{1}$ and $x_{9}$, and that for these variables, membership functions exist for the conditions low, medium, and high as well as low, and high, respectively, the following antecedents are derived:

$$
\begin{gathered}
R A_{A, 1}: \quad x_{1}=\text { low AND } x_{9}=\text { low } \\
R A_{A, 2}: \quad x_{1}=\text { low AND } x_{9}=\text { high } \\
R A_{A, 3}: \quad x_{1}=\text { medium AND } x_{9}=\text { low } \\
R A_{A, 4}: \quad x_{1}=\text { medium AND } x_{9}=\text { high } \\
R A_{A, 5}: \quad x_{1}=\text { high AND } x_{9}=\text { low } \\
R A_{A, 6}: \quad x_{1}=\text { high AND } x_{9}=\text { high }
\end{gathered}
$$

where $A$ stands for the Achiever class.

\section{Step 3: Derive rule consequences}

Now that a set of antecedents is known for each HEXAD class, the consequences that accompany these antecedents to form a set of fuzzy IF-THEN rules may be derived. Unlike the antecedents, these consequences are 'learned' from the data available, rather than 'suggested' by experts. It is assumed that a consequence should be a linear function of the input parameters of the rule's antecedents - i.e., a function of the input parameters that were mapped onto a specific HEXAD class - as is often the case in TakagiSugeno systems. Hence, building on the example that was given in the previous Step, the rules to be derived 
may have the form of:

$$
\begin{aligned}
& R_{A, 1}: \quad \text { IF } x_{1}=\text { low AND } x_{9}=\text { low } \quad \text { THEN } \\
& \text { Achiever }=\theta_{A, 1, \varepsilon}+\theta_{A, 1,1} \cdot x_{1}+\theta_{A, 1,2} \cdot x_{9} \\
& R_{A, 2} \text { : IF } x_{1}=\text { low AND } x_{9}=\text { high THEN } \\
& \text { Achiever }=\theta_{A, 2, \varepsilon}+\theta_{A, 2,1} \cdot x_{1}+\theta_{A, 2,2} \cdot x_{9} \\
& R_{A, 6}: \quad \text { IF } x_{1}=\text { high AND } x_{9}=\text { high } \quad \text { THEN } \\
& \text { Achiever }=\theta_{A, 6, \varepsilon}+\theta_{A, 6,1} \cdot x_{1}+\theta_{A, 6,2} \cdot x_{9},
\end{aligned}
$$

where again, $A$ stands for the Achiever class.

To be able to learn the optimal consequence (i.e., the $\theta$ 's) that may be implied by the suggested antecedents and the actual supervised data available, a number of linear inequalities is solved. In this linear program, a pseudo-inverse solver determines the optimal consequence parameters, given a subject's actual values on suggested input parameters and the subject's actual degree of belonging to a particular HEXAD class. Since the optimal consequence parameters are estimated based on a subject's actual degree of belonging, this Step - and all consecutive Steps - are performed using the supervised dataset, which includes campaign participants that completed the post-test suvey, and hence, for whom estimates of their actual degree of belonging to each of the HEXAD classes are known. Summarizing, the following linear program is solved:

$$
\hat{\mathbf{Y}}_{o}=\mathbf{Y}_{o}
$$

where $\mathbf{Y}_{o}$ is the vector of actual degree of belonging $y_{s, o}$ to a particular HEXAD class $o$ for a particular subject $s$, and where $\hat{\mathbf{Y}}_{o}$ is the vector of predictions:

$$
\hat{y}_{s, o}=\sum_{j=1}^{N} \hat{\beta}_{s, o, j} \cdot\left(\theta_{o, j, \varepsilon}+\sum_{i \in \boldsymbol{R} \boldsymbol{V}_{o}} \theta_{o, j, i} \cdot x_{s, i}\right)
$$

for a particular HEXAD class $o$ for a particular subject $s$. In $\hat{Y}_{o}, j=1 \ldots N$ is the rule number for the rule base of class $o$, and $i \in \boldsymbol{R} \boldsymbol{V}_{\boldsymbol{o}}$ are the input parameters mapped onto this class $o$. Then, $\hat{\beta}_{s, o, j}$ is the normalized degree of applicability of rule $j$ in the rule base for HEXAD class $o$ for a particular subject $s$, defined as: $\beta_{s, o, j} / \sum_{j=1}^{N} \beta_{s, o, j}$, and $x_{s, i}$ are the actual parameter values for input parameter $i$ of a particular subject $s$. Then, $\theta_{o, j, i}$ and $\theta_{o, j, \varepsilon}$ are the consequence parameters that need to be estimated, where $\theta_{o, j, \varepsilon}$ represents the error term of the linear function. Finally, in $\hat{\beta}_{s, o, j}$, $\beta_{s, o, j}$ is defined as:

$$
\beta_{s, o, j}=\min _{\forall i \in \boldsymbol{R} \boldsymbol{V}_{o}} \mu_{c_{i}}\left(x_{s, i}\right),
$$

where $\mu_{c_{i}}$ is the membership function - as derived in Step 1 - of the condition $c_{i}$ for input parameter $i$, for the actual value $x_{s, i}$ of input parameter $i$ for subject $s$, where $i \in \boldsymbol{R} \boldsymbol{V}_{\boldsymbol{o}}$. In Step 3, a potential rule is omitted and therefore deleted from the rule base of a particular HEXAD class $o$, if the degree of applicability $\beta_{s, o, j}$ is 0 for each subject $s$ in the dataset.

If the example that was given in the previous Step is considered again, the following set of linear inequalities is solved to learn the optimal consequence parameters (i.e., the $\theta$ 's):

$$
\left[\begin{array}{c}
\sum_{j=1}^{6} \hat{\beta}_{1, A, j} \cdot\left(\theta_{A, j, \varepsilon}+\sum_{i \in \boldsymbol{R} \boldsymbol{V}_{\boldsymbol{A}}} \theta_{A, j, i} \cdot x_{1, i}\right) \\
\sum_{j=1}^{6} \hat{\beta}_{2, A, j} \cdot\left(\theta_{A, j, \varepsilon}+\sum_{i \in \boldsymbol{R} \boldsymbol{V}_{\boldsymbol{A}}} \theta_{A, j, i} \cdot x_{2, i}\right) \\
\vdots \\
\sum_{j=1}^{6} \hat{\beta}_{46, A, j} \cdot\left(\theta_{A, j, \varepsilon}+\sum_{i \in \boldsymbol{R} \boldsymbol{V}_{\boldsymbol{A}}} \theta_{A, j, i} \cdot x_{46, i}\right)
\end{array}\right]=\left[\begin{array}{c}
y_{1, A} \\
y_{2, A} \\
\vdots \\
y_{46, A}
\end{array}\right],
$$

where again, $A$ stands for the Achiever class.

\section{Step 4: Evaluate performance}

To evaluate the performance of the set of rules in predicting a subject's degree of belonging to a particular HEXAD class, the Sum of Squared Errors (SSE) is determined (i.e., both per class and in total).

Additionally, the accuracy of a predicted degree of belonging to a HEXAD class is evaluated, by ranking the predicted classes and comparing the actual class a subject belongs to most, with the predicted class a subject belongs to most. Furthermore, it is also evaluated whether a subject's most representative predicted class is among the actual top three classes that best represent the subject.

\subsection{Model validation}

To obtain a valid estimate of the performance measures, Step 3 and Step 4 should be performed on different subsamples of the supervised dataset (i.e., a train and test set respectively).

To validate the estimate of the performance measures, a five-fold cross-validation was performed, see Figure 2. In five-fold cross-validation, the original sample is randomly partitioned into five equal sized subsamples. Of the five subsamples, a single subsample $(20 \%$ of data) is retained as the validation data for testing the model, and the remaining four subsamples ( $80 \%$ of data) are used as training data. The cross-validation process is then repeated five times, with each of the five subsamples used exactly once as the validation data. 


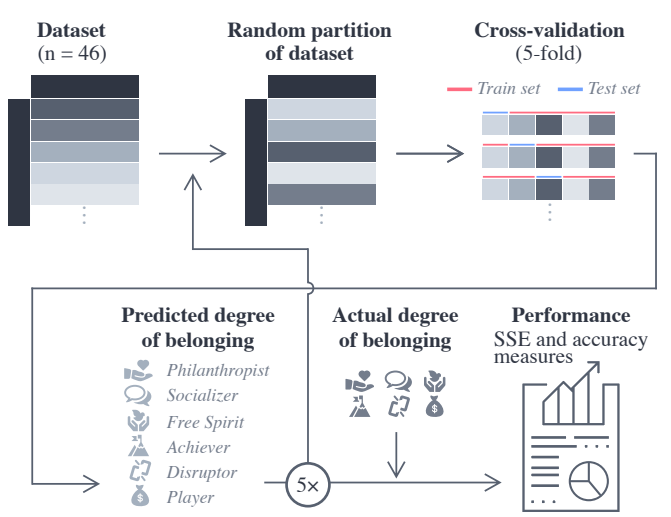

Figure 2: Model validation process.

The five results can then be averaged to produce a single estimation. The advantage of this method is that all observations are used for both training and validation, and each observation is used for validation exactly once. Finally, the cross-validation process was performed five times, to allow for different random partitions of the dataset. Results on performance measures have been averaged.

\section{Results}

\subsection{Results of data collection}

Unsupervised user event data from the mHealth tool was collected for 146 participants. Nevertheless, only 48 subjects have completed the post-test questionnaire, which was used to establish a degree of belonging to each HEXAD class. Of this sample of 48 , only 46 samples were eligible to be included in the supervised dataset after cleaning of ambiguous entries. Figure 3 depicts the distribution of the HEXAD classes in which the participants achieved respectively the highest (and resp. lowest) survey score.



Figure 3: Distribution of subject's HEXAD classes.

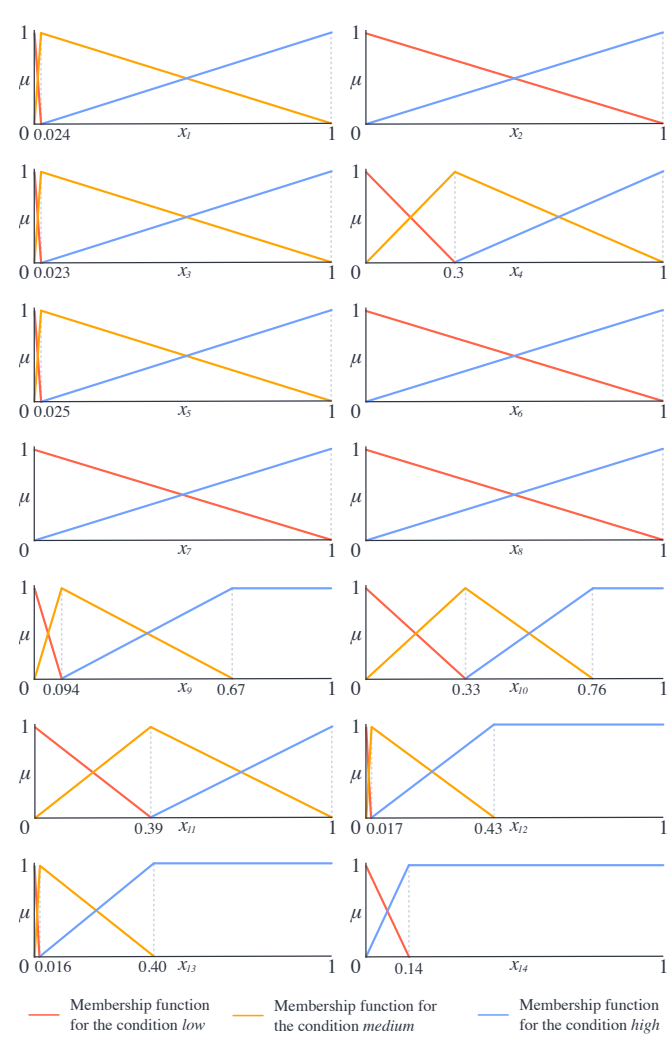

Figure 4: Defined membership functions $\mu_{c_{i}}$ for each of the 14 input parameters.

\subsection{Derivation of membership functions and fuzzy rule bases}

Triangular membership functions were determined for the 14 input parameters based on the unsupervised dataset, see Figure 4. Subsequently, to establish the sets of fuzzy rules that describe a particular HEXAD class, it was necessary to obtain a mapping between input parameters and classes, based on the input's (expected) predictive power of a certain class. From an expert session the following mapping of inputs to classes was obtained: one's degree of belonging to the Philanthropist class may be well explained by input parameters $x_{6}, x_{9}, x_{11}$, and $x_{14}$, which may be indicators of altruistic behaviors, such as caring for the well-being of others; one's degree of belonging to the Socialiser class may be well explained by the input parameters $x_{6}, x_{7}$, and $x_{8}$, which are all indicators of interaction with others; one's degree of belonging to the Free Spirit class may be well explained by the input parameters $x_{3}$, and $x_{4}$, which are indicators of autonomy and self-expression; one's degree of belonging to the Achiever class may be well explained by the input parameters $x_{1}, x_{3}, x_{9}$, and $x_{10}$, which are indicators of pursuing mastery; one's degree of belonging to the Disruptor class may be well explained by the input parameter $x_{5}$, which may be an indicator of cheating, 
and therefore disruptive behavior; one's degree of belonging to the Player class may be well explained by the input parameters $x_{2}$, and $x_{9}$, which are indicators of pursuing (extrinsic) rewards.

From this mapping, rule antecedents and rule consequences are derived as described in Step 2 and Step 3 of Section 3.3. For the Philanthropist class theoretically 108 rules could have been derived based on this mapping. However, rules that never fire for any subject in the training set are eventually omitted. Since this cleaning of the rule bases is dependent on the observations that are actually included in the training set, different rules may have been omitted throughout cross-validation (where multiple instances of training sets and test sets have been evaluated). It was found that, on average, only 91.36 rules (out of a theoretical maximum of 108 rules) were included in the rule base for the Philanthropist class. Similarly it was found that for the Socialiser, Free Spirit, Achiever, Disruptor, and Player class 6.8 out of $8,7.8$ out of $9,62.6$ out of 81,3 out of 3 , and 6 out of 6 rules were included on average, respectively. Randomly chosen instances of these rules were:

$R_{A, 1}: \quad$ IF $x_{1}=$ low AND $x_{3}=$ low AND $x_{9}=$ low AND $x_{10}=$ medium $\quad$ THEN Achiever $=0.199-$ $0.000221 \cdot x_{1}-0.00111 \cdot x_{3}-0.00976 \cdot x_{9}+0.169 \cdot x_{10}$ $R_{A, 20}: \quad$ IF $x_{1}=$ low AND $x_{3}=$ high AND $x_{9}=$ medium AND $x_{10}=$ low $\quad$ THEN Achiever $=$ $0.175+0.00349 \cdot x_{1}+0.00786 \cdot x_{3}+0.0124 \cdot x_{9}+0.0549 \cdot x_{10}$,

where again, $A$ stands for the Achiever class, and:

$$
\begin{gathered}
R_{S, 1}: \quad \text { IF } x_{6}=\text { high AND } x_{7}=\text { medium AND } \\
x_{8}=h i g h \quad \text { THEN Socialiser }= \\
0.283+0.283 \cdot x_{6}+0.0314 \cdot x_{7}+0.283 \cdot x_{8}
\end{gathered}
$$

where $S$ stands for the Socialiser class.

\subsection{Evaluation of model performance}

Repeated five-fold cross-validation reports an average SSE of $31.576(\sigma=26.640)$. The average SSE for the HEXAD classes Philanthropist, Socialiser, Free Spirit, Achiever, Disruptor and Player are 6.516, 7.087, 6.216, 2.207, 1.167, and 8.393, respectively. Furthermore, the model obtained an average accuracy on correctly predicting a user's actual most representative class of $0.200(\sigma=0.130)$, while obtaining an average accuracy on correctly predicting one of the user's top-three most representative classes of $0.622(\sigma=0.137)$.

\section{Discussion and Conclusions}

From the SSE per HEXAD class it can be derived that especially the degree of belonging to the Achiever and
Disruptor class (SSE of 2.207 and 1.167, respectively) are predicted with a higher precision than the subject's degree of belonging to either of the four other classes. The mHealth tool under investigation seems to particularly cater to the needs of Achievers, since the configuration of GameBus that was evaluated was mainly centered around challenges and competition, and therefore 'striving behavior' may be effectively captured by the input parameters mapped onto this HEXAD class, i.e., $x_{1}, x_{3}, x_{9}$, and $x_{10}$. Additionally, it seems that Disruptive behavior is not really supported by the currently evaluated configuration of the mHealth tool, and can therefore most probably not reliably be explained by input parameter $x_{5}$. Nevertheless, the prediction error (i.e., SSE) is lowest for this class. Besides the unexpected predictive power of input parameter $x_{5}$ explaining this result, this results is more likely explained by the fact that only a few subjects reported to identify themselves with the Disruptor class in the first place (i.e., $58 \%$ of post-tested subjects reported that they related to the Disruptor class least, whereas only $4 \%$ indicated that this class represented them best, also see Figure 3.

Finally, from the analysis of accuracy scores for the entire fuzzy model, it may be derived that user preferences may actually be understood from user event data in an mHealth setting. A truly random predictor may correctly predict the class that represents a subject most with a probability of $1 / 6=0.17$. The fuzzy model obtained an accuracy of 0.200 on average, which is slightly better. Similarly, the probability that the most probable predicted class of a truly random predictor is among the top three actual classes that most represent a subject is $3 / 6=0.5$. Again, the fuzzy model obtained an accuracy (0.622) that is slightly better than a random system may achieve.

Understanding user preferences provides a basis for personalization of GameBus' content: based on one's degree of belonging to a specific HEXAD class, the user may be confronted for example with personally tailored challenges or custom supportive messages, in order to foster engagement and thereby sustain the impact of the mHealth intervention in the long run.

\subsection{Study limitations}

As outlined in Section 3.1, for a scientific experiment, some participants were confronted with a personal goal of obtaining 50 virtual points, while others were not. Of the sample of 46 post-tested subjects, 9 participants were never given a goal of obtaining 50 virtual points. Therefore, particularly input parameter $x_{2}$ may have been biased, which may explain why the Player class could not be reliably predicted (i.e., for this class, the SSE was highest). In future analyses, these treatment 
effects may be accounted for. Moreover, it may be that the mHealth tool did not fully cater to all preferences and needs a certain HEXAD class may require. Therefore, the input parameters available may have lacked predictive power for some of the classes.

\subsection{Future work}

To better estimate performance measures and to strengthen the results obtained, future research should focus on collecting additional data. Moreover, future work may focus on learning also the rule antecedents (i.e., the set of relevant input parameters $\boldsymbol{R} \boldsymbol{V}_{\boldsymbol{o}}$ for a particular HEXAD class $o$ ) from data, instead of only the consequence parameters (i.e., the $\theta$ 's). Additionally, it would make sense to apply some further rule base simplification an pruning methods to reduce the number of rules per inference system, for example using the rule base simplification procedure by Setnes and colleagues 13. Finally, the performance of the modeling approach adopted in this study should be compared to other (fuzzy) modeling approaches.

\section{Acknowledgement}

This work is partly funded by the research project GOAL (443001101), from the Netherlands Organisation for Health Research and Development (ZonMw).

\section{References}

[1] R. Bartle, Hearts, clubs, diamonds, spades: Players who suit MUDs, Journal of Virtual Environments 1 (1) (1996).

[2] J. M. Costa Sousa, da, U. Kaymak, Fuzzy decision making in modeling and control, Vol. 27 of World Scientific series in robotics and intelligent systems, World Scientific, Singapore, 2002.

[3] A. DeSmet, D. Van Ryckeghem, S. Compernolle, T. Baranowski, D. Thompson, G. Crombez, K. Poels, W. Van Lippevelde, S. Bastiaensens, K. Van Cleemput, et al., A meta-analysis of serious digital games for healthy lifestyle promotion, Preventive medicine 69 (2014) 95-107.

[4] S. Deterding, D. Dixon, R. Khaled, L. Nacke, From game design elements to gamefulness: Defining gamification, in: Proceedings of the 15th International Academic MindTrek Conference, Vol. 11, 2011, pp. 9-15.

[5] P. Dinesh Asanka, A. Perera, Defining fuzzy membership function using box plot, International Journal of Research in Computer Applications and Robotics 5 (11) (2017) 1-10.
[6] L. V. Eckerstorfer, N. K. Tanzer, C. VogrincicHaselbacher, G. Kedia, H. Brohmer, I. Dinslaken, K. Corcoran, Key elements of mhealth interventions to successfully increase physical activity: Meta-regression, JMIR mHealth and uHealth 6 (11) (2018) .

[7] E. A. Finkelstein, B. A. Haaland, M. Bilger, A. Sahasranaman, R. A. Sloan, E. E. K. Nang, K. R. Evenson, Effectiveness of activity trackers with and without incentives to increase physical activity (trippa): a randomised controlled trial, The lancet Diabetes \& endocrinology 4 (12) (2016) 983-995.

[8] L. R. Goldberg, An alternative "description of personality": the big-five factor structure, Journal of Personality and Social Psychology 59 (6) (1990) 1216-1229.

[9] J. Hamari, J. Koivisto, H. Sarsa, Does gamification work?-a literature review of empirical studies on gamification, in: 2014 47th Hawaii international conference on system sciences (HICSS), IEEE, 2014, pp. 3025-3034.

[10] A. J. Lentferink, H. K. Oldenhuis, M. de Groot, L. Polstra, H. Velthuijsen, J. E. van GemertPijnen, Key components in ehealth interventions combining self-tracking and persuasive ecoaching to promote a healthier lifestyle: A scoping review, J Med Internet Res 19 (8) (2017) e277.

[11] A. Marczewski, Gamification, game thinking \& motivational design, in: Even Ninja Monkeys Like to Play, CreateSpace Independent Publishing Platform, 2015, pp. 69-84.

[12] R. M. Ryan, E. L. Deci, Self-determination theory and the facilitation of intrinsic motivation, social development, and well-being., American psychologist 55 (1) (2000) 68-78.

[13] M. Setnes, R. Babuska, U. Kaymak, H. R. van Nauta Lemke, Similarity measures in fuzzy rule base simplification, IEEE Transactions on Systems, Man, and Cybernetics, Part B (Cybernetics) 28 (3) (1998) 376-386.

[14] G. F. Tondello, R. R. Wehbe, L. Diamond, M. Busch, A. Marczewski, L. E. Nacke, The gamification user types HEXAD scale, in: Proceedings of the annual symposium on computer-human interaction in play, ACM, 2016, pp. 229-243.

[15] L. Zadeh, Fuzzy sets, Information and Control 8 (3) (1965) $338-353$. 